\title{
Aerodynamic and Aeroelastic Tool for Wind Turbine Applications
}

\section{Valerio Viti*}

ANSYS, Inc., Lebanon, NH, 03766, USA

\section{Giuliano Coppotelli** and Federico de Pompeis***}

University of Rome "La Sapienza", Rome, Italy

\author{
Pier Marzocca**** \\ Clarkson University, Potsdam, NY, USA
}

\begin{abstract}
The present work focuses on the unsteady aerodynamics and aeroelastic properties of a small-medium sized wind-turbine blade operating under ideal conditions. A tapered/twisted blade representative of commercial blades used in an experiment setup at the National Renewable Energy Laboratory is considered. The aerodynamic loads are computed using Computational Fluid Dynamics (CFD) techniques. For this purpose, FLUENT ${ }^{\circledR}$, a commercial finite-volume code that solves the Navier-Stokes and the Reynolds-Averaged Navier-Stokes (RANS) equations, is used. Turbulence effects in the 2D simulations are modeled using the Wilcox $k$ - $w$ model for validation of the CFD approach. For the 3D aerodynamic simulations, in a first approximation, and considering that the intent is to present a methodology and workflow philosophy more than highly accurate turbulent simulations, the unsteady laminar Navier-Stokes equations were used to determine the unsteady loads acting on the blades. Five different blade pitch angles were considered and their aerodynamic performance compared. The structural dynamics of the flexible wind-turbine blade undergoing significant elastic displacements has been described by a nonlinear flap-lag-torsion slender-beam differential model. The aerodynamic quasi-steady forcing terms needed for the aeroelastic governing equations have been predicted through a strip-theory based on a simple 2D model, and the pertinent aerodynamic coefficients and the distribution over the blade span of the induced velocity derived using CFD. The resulting unsteady hub loads are achieved by a first space integration of the aeroelastic equations by applying the Galerkin's approach and by a time integration using a harmonic balance scheme. Comparison among two- and three- dimensional computations for the unsteady aerodynamic load, the flap, lag and torsional deflections, forces and moments are presented in the paper. Results, discussions and pertinent conclusions are outlined.
\end{abstract}

Key words: Wind turbine, aeroelasticity, aerodynamics, CFD

\section{Nomenclature}

$A$
$a$
$a$
$C_{p}$
$C_{d}$
$C_{l}$
$C_{x}$

amplitude of oscillation

cylinder diameter

lift curve slope

pressure coefficient

drag coefficient

lift coefficient

force coefficient in the $\mathrm{x}$ direction

This is an Open Access article distributed under the terms of the Creative Commons Attribution Non-Commercial License (http://creativecommons.org/licenses/by$\mathrm{nc} / 3.0 /$ which permits unrestricted non-commercial use, distribution, and reproduction in any medium, provided the original work is properly cited. (c)

* Senior Engineer, Corresponding author: Valerio.viti@ansys.com

** Assistant Professor

*** Graduate Student

$* * * *$ Associate Professor 


$\begin{array}{ll}\boldsymbol{C}_{\boldsymbol{y}} & \text { force coefficient in the y direction } \\ \boldsymbol{c} & \text { chord } \\ \boldsymbol{d} \boldsymbol{t} & \text { time step } \\ \boldsymbol{F}_{\boldsymbol{x}} & \text { X component of the resultant pressure force acting on the vehicle } \\ \boldsymbol{F}_{\boldsymbol{y}} & \text { Y component of the resultant pressure force acting on the vehicle } \\ \boldsymbol{f}, \boldsymbol{g} & \text { generic functions } \\ \boldsymbol{h} & \text { height } \\ \boldsymbol{N}, \boldsymbol{N}_{\boldsymbol{b}} & \text { Number of blades of rotor } \\ \boldsymbol{r} & \text { Radial distance from axis of rotation } \\ \boldsymbol{R} & \text { Radius of rotor } \\ \boldsymbol{V}_{t o t} & \text { Resultant total velocity, } \\ \boldsymbol{V}_{\infty} & \text { Freestream velocity } \\ \boldsymbol{x} & \text { Dimensionless local spanwise direction } \\ \boldsymbol{S}_{\boldsymbol{x}}, \boldsymbol{S}_{\boldsymbol{r}}, \boldsymbol{S}_{z} & \text { Drag, radial, and vertical shear forces at the rotor hub, respectively } \\ \boldsymbol{N}_{F}, \boldsymbol{N}_{L} & \text { Root bending and torque moments, respectively } \\ \boldsymbol{T} ; \boldsymbol{H} ; \boldsymbol{Y} & \text { Total forces in the non-rotating frame for a N bladed windmill, respectively } \\ \boldsymbol{M}_{\boldsymbol{x}} ; \boldsymbol{M}_{\boldsymbol{y}} ; \boldsymbol{Q} & \text { Total moments in the non-rotating frame for a N bladed windmill, respectively }\end{array}$

\title{
Subscripts
}

\section{$i$}

$r$

$\infty$

\section{Superscript}

$2 D$

$3 D$

\section{Greek Symbols}

induced

radial distance from axis of rotation

freestream conditions

two-dimensional

three-dimensional

\author{
angle-of-attack \\ inflow ratio \\ blade twist \\ angular velocity of rotor \\ trailing-edge (TE) non-dimensional angular deflection rate \\ azimuthal position of the blade
}

\section{Introduction}

Wind turbines operate in an aerodynamic environment which is difficult to analyze and predict. The periodic and aperiodic unsteadiness of the atmospheric boundary layer makes the design of wind-energy systems very difficult since their design is typically optimized for steady state conditions. In particular, the performance of the turbine blades is strongly affected by the stochastic nature of the airloads; the blade structure needs to be built for the best efficiency in converting the wind energy into usable mechanical energy while maintaining structural integrity [1-4]. The need to account for the random nature of the airloads leads to heavier, stiffer blade designs that are not optimized for the prevailing operating conditions. Also, the heavier blades limit the rotational speed of the rotor as the inertial forces acting at the root of the blades are too high for safe operation. The less-than-optimal design of the blades leads to higher initial and operating costs since centrifugal and gravity loads are primarily responsible for fatigue failure 
[1-4]. These design weaknesses are contributing factors to the competitive disadvantage of wind energy against other forms of renewable and non-renewable energy sources.

Options to improve the efficiency of wind turbine blades are varied. On one side of the spectrum there is the option to design lighter, stronger blades using more advanced materials. However, the high costs associated with the advanced structure and materials make this option economically not viable on the opposite side of the spectrum is the implementation of an active control system that, rather than modifying the blade structure to make it stronger and stiffer, allows the blade to morph and to change its shape in order to alleviate the instantaneous loads. By using this control strategy, the blade structural loads can be reduced and the operation of the wind-turbine can be continued under adverse environmental conditions. An additional advantage of an active control system is the effective stabilization of the blade which protects the wind turbine system against aeroelastic instabilities $[5,6]$.

However, the proposed control system concept needs development and verification. In order to achieve these goals, an aeroelastic model that couples the blade structural response to the unsteady aerodynamic loads is being developed. In order to validate the control system as a proofof-concept, the aeroelastic model needs to be able to capture the characteristic behavior of both the fluids and structural domains and yet be relatively low-cost for quick turnaround design iterations.

One of the bottlenecks in the development of the aeroelastic model is how to best capture the aerodynamic behavior of the blade since such a prediction is critical to the reliable description of the system. Often aerodynamic modeling has been accomplished by means of engineeringtype empirical models like the ONERA or the BeddoesLeishman models $[7,8]$. The airloads models are based on the conventional rotorcraft lifting line approach of treating each $3 \mathrm{D}$ rotor blade as a series of $2 \mathrm{D}$ airfoil sections and calculating the section airloads based on local section aerodynamic parameters. This is mainly due to the fact that the considered blades have large aspect ratio. In addition, the aerodynamic loads are based on the blade element momentum theory which assumes a 2D flow over each blade airfoil section. The induced velocity is obtained from the simple momentum theory, generalized dynamic wake, prescribed wake, or free wake. Static stall and compressibility effects are implicitly included in tabulated data as functions of angle-of-attack. Another assumption is that the quasi-steady aerodynamics governs the reverse flow region. Navier-Stokes solvers have been usually restricted to two-dimensional analysis and only a few attempts have been made to advance the state-of-the- art in aeroelasticity from two-dimensional to quasi-threedimensional and fully three-dimensional Navier-Stokes solutions $[9,10]$.

In this paper, the aerodynamic loads are computed using Computational Fluid Dynamics (CFD) techniques. A commercial finite-volume code, FLUENT $^{\circledR}$, that solves the Navier-Stokes and Reynolds-Averaged Navier-Stokes (RANS) equations is used. Turbulence effects, considered only in the 2D simulations, are modeled using Wilcox's $k$ - $w$ model [10]. For the 3D simulations, the unsteady laminar Navier-Stokes equations were used to compute the unsteady periodic loads. Moreover, the structural dynamics of the flexible wind-turbine blade undergoing significant elastic displacements has been described by the nonlinear flaplag-torsion slender-beam differential model introduced by Hodges and Dowell [11]. The aerodynamic quasi-steady forcing terms needed for the aeroelastic governing equations have been predicted through a strip-theory based on a simple 2D model [11-13] whose pertinent aerodynamic coefficients could be derived from Computational Fluid Dynamics (CFD) techniques [14]. The resulting unsteady hub loads are finally achieved by a first space integration of the aeroelastic equations by applying the Galerkin approach - using as shape functions the bending and torsion free vibration eigenmodes of a uniform cantilever beam - and then in time using the harmonic balance approach $[11,15,16]$. Referring to the three-bladed wind turbine used for the experimental test at the National Renewable Energy Laboratory (NREL) $[17,18]$ represented in Fig. 1, the goals of this paper are twofold: i) to develop an aerodynamic and aeroelastic tool for wind turbine design based on the fully-threedimensional Navier-Stokes equations, and ii) to highlight the differences and deficiencies of a two-dimensional versus a three-dimensional wind turbine aerodynamic modeling.

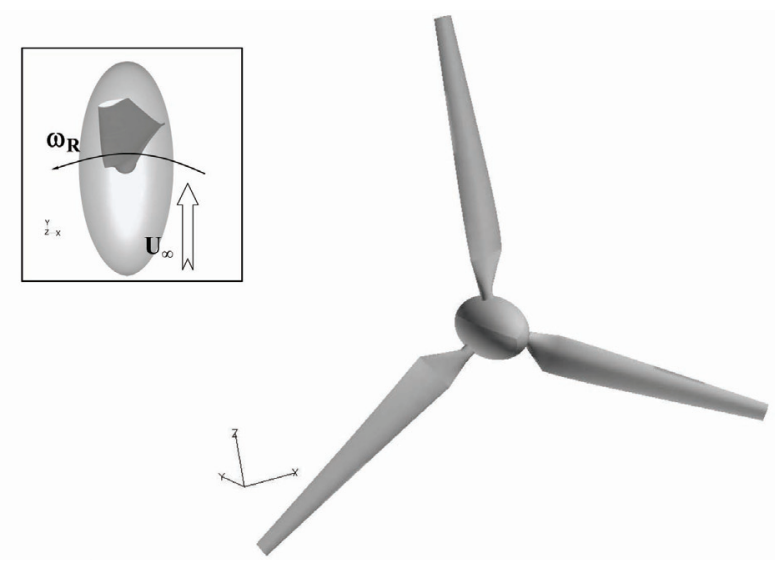

Fig. 1. Isometric view of the three-bladed rotor $\$ 809$ airfoil. The insert shows the orientation of the axes of reference. 
Desired outputs from this numerical tool are the vibration levels due to the unsteady loads acting on the rotating wind-turbine blades, and the prediction of the fatigue-life of the structure and the exterior noise of the operating wind turbines. Ultimately this effort is meant to provide the wind turbine industry with a tool for the design of more efficient wind turbines.

\section{Computational Fluid Dynamics Model}

The CFD model of the blade was developed using a commercial CFD code, Fluent 6.2 [14]. Fluent was chosen due to its validated performance and availability of physical models and widespread presence in the power generation industry. The blade geometry was reconstructed in the mesh generator, Gambit 2.3, from the data provided in Giguère and Selig [17]. The 3D simulations were run using the fully 3D unsteady laminar Navier-Stokes (NS) equations. Note that while the flow is turbulent, unsteady laminar simulations were used in consideration of the fact that the present work presents a methodology and develops a tool for the aeroelastic analysis of slender wind turbine blades which is independent of the unsteady nature of the flowfield. That is, the same methodology and workflow philosophy that can be applied to a laminar flow can also be used for a turbulent flow, and this flexibility is part of the appeal of the present method. The intent here is to provide the reader with a recipe on the procedure to follow in wind turbine rotor fluid-structure simulations, which has a general value, independently of the qualitative nature of the CFD simulations. In future work the extension to a turbulent fluid model will be presented. The 2D simulations were run using the unsteady ReynoldsAveraged Navier-Stokes (URANS) equations and turbulence effects were computed using the Wilcox $k-w$ turbulence model $[10,14]$. In both cases the flow was considered to be

Table 1. Blade and Rotor dimensions

\begin{tabular}{|l|l|}
\hline \hline Parameters & \\
\hline \hline Blade Span (axis of revolution-tip), & $5.532 \mathrm{~m}$ \\
Blade Span (junction-tip), $\mathrm{r}_{100}$ & $5.024 \mathrm{~m}$ \\
Blade Span (root-tip), $\mathrm{r}_{\mathrm{B}}$ & $4.275 \mathrm{~m}$ \\
${\text { Root Chord, } \mathrm{c}_{\mathrm{root}}}^{\text {Tip Chord, } \mathrm{c}_{\text {tip }}}$ & $0.737 \mathrm{~m}$ \\
Average Chord, $\mathrm{C}_{\text {Avg }}$ & $0.305 \mathrm{~m}$ \\
Average Blade Twist (root-to-chord) & $-5 \mathrm{deg} / \mathrm{m}$ \\
Number of Blades, N & 3 \\
Rotor Swept Area & $96.17 \mathrm{~m}^{2}$ \\
Rotor Solidity & 0.069 \\
\hline \hline
\end{tabular}

incompressible and a pressure-based solver was employed. Second-order upwind spatial discretization was used for the transport equation and a second-order Runge-Kutta scheme was used for time discretization [14].

\subsection{Boundary conditions}

The geometry of the wind turbine and the definition of the boundary conditions with a uniform velocity inlet profile, allows the use of rotationally periodic boundary conditions so that only one single blade has been simulated. The blade geometry is shown schematically in Fig. 1 and the blade dimensions are summarized in Table 1. The blade twist is plotted together with the nominal angle-of-attack for a rotational speed of $72 \mathrm{rpm}$ in Fig. 2.

Additional geometrical parameters are provided in the numerical results, Section 4. The computational domain, shown in Fig. 3, comprises a section of a cylinder $120^{\circ}$ in arc. The radial dimension is $30 \mathrm{~m}$ and the domain extends $15 \mathrm{~m}$ in front and aft of the rotor so as to minimize any influence from the boundary conditions. The operating conditions for the simulations are summarized in Table 2. A uniform steady velocity profile of $6.0 \mathrm{~m} / \mathrm{s}$ was used for the upstream boundary condition while a constant-pressure boundary condition was used for the downstream boundary condition

Table 2. Summary of the flow conditions, ISA sea-level

\begin{tabular}{|l|l|}
\hline \hline Parameter & Value \\
\hline \hline $\mathrm{Pa}_{\infty}$ & $101,325 \mathrm{~Pa}$ \\
$\mathrm{~T}_{\infty}$ & $293 \mathrm{~K}$ \\
$\mathrm{~V}_{\infty}$ & $6 \mathrm{~m} / \mathrm{s}$ \\
$\omega_{\mathrm{R}}$ & $72 \mathrm{rpm}$ \\
$\mathrm{V}_{\text {Tot, tip }}$ & $42.1 \mathrm{~m} / \mathrm{s}$ \\
$\mathrm{Ma}_{\text {Tot, tip }}$ & 0.12 \\
Tip-speed & 7.0 \\
\hline \hline
\end{tabular}

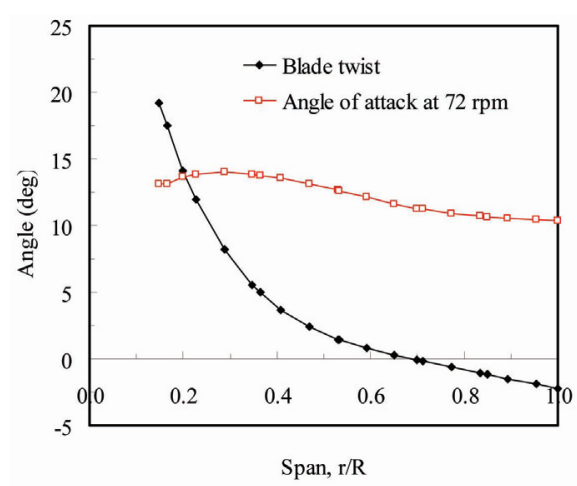

Fig. 2. Twist and nominal angle of attack distribution of the blade for a pitch angle of 0 degree. The nominal blade pitch angle is given by the twist angle at $r / R=0.75$. 
and for the side at the plane of maximum radius. An axis boundary condition was used for the axis of rotation of the rotor and rotationally-periodic boundary conditions were applied at the two side planes.

\subsection{Computational grid}

The computational mesh is a hybrid mesh that contains both hexahedral and tetrahedral elements for a total of $1.14 \mathrm{M}$ cells. The hexahedral elements are primarily used to construct a finer mesh on the solid surfaces of the turbine so as to capture the viscous effects of the boundary layer. A canonical grid convergence study was not performed for the present work although solution-steered grid adaption was performed to study the sensitivity of the force and momentum results to mesh refinement which was found to be minor for the present conditions.

\subsection{D/3D Aerodynamic coupling}

In this work $2 \mathrm{D}$ and a $3 \mathrm{D}$ aerodynamic models have been compared. The $2 \mathrm{D}$ model enables one to calculate the inflow ratio, denoted as $\lambda_{i}^{2 D}$, through a combined blade-element and momentum theory. It is possible to write $[15,23]$

$$
\lambda_{i}^{2 D}(x)=\frac{v_{i}}{\Omega x}=-\left(\frac{c_{l \alpha} \sigma}{16}-\frac{\lambda_{c}}{2}\right)+\sqrt{\left(\frac{c_{l \alpha} \sigma}{16}-\frac{\lambda_{c}}{2}\right)^{2}+\frac{c_{l \alpha} \sigma}{8}\left(\theta_{t w}+\lambda_{c}\right)}
$$

where $c_{l \alpha}=2 \pi ; \sigma=\frac{N_{b} c}{\pi R}$ (see Table 1$)$;

$N_{b}=3 ; \lambda_{c}=\frac{V_{\infty}}{\Omega R} ; x=\frac{r}{R} ; \theta_{t w}=f(x)$.

In the previous equation, $\lambda_{i}^{2 D}$ is a function of the local spanwise distance $x$. The evaluation of the inflow ratio is one of the critical issues when dealing with the aeroelastic response of the rotating blade. In this paper, the $3 \mathrm{D}$ aerodynamic computations have been used to estimate the actual inflow ratio. Indeed, the results obtained from the CFD computations have been used in the same way the experimental aerodynamicist would use if the equivalent $2 \mathrm{D}$ coefficients have to be estimated from in-flight or wind tunnel tests. This approach has the great advantage in reducing the time required for the aeroelastic computations since the time consuming CFD simulations are run only once. Following [15] and [23], the inflow ratio, as function of the spanwise location $\lambda_{i}^{3 D}(x)$, could be estimated from the knowledge of the effective angle of attack gained from the $3 \mathrm{D}$ CFD. The sequence to evaluate the inflow ratio for the $3 \mathrm{D}$ case is as follows:

$$
\begin{aligned}
& \alpha_{e f f}(x)=\theta_{t w}-\tan ^{-1}\left(\frac{V_{\infty}-\lambda_{i}^{2 D} \Omega R}{\Omega R}\right) \\
& \lambda_{i}^{3 D}=c_{1} \alpha_{e f f}+c_{2}
\end{aligned}
$$

$$
\lambda_{i}^{3 D}-\lambda_{i}^{2 D} \leq \varepsilon
$$

First, the effective angle of attack, $\alpha_{e f f}$, is estimated from Eq. (2a) considering the $2 \mathrm{D}$ inflow ratio as the starting point, then $\lambda_{i}^{3 D}$ is evaluated from Eq. (2b). At this point, the values of $\lambda_{i}^{2 D}$ and $\lambda_{i}^{3 D}$ are different, therefore, Eqs. (2a) and (2b) are iteratively solved until Eq. (2c) is satisfied. Note that $\varepsilon$ is a small arbitrary quantity and for the present simulations it was set to $1 \times 10^{-5}$. The coefficients $c_{1}$ and $c_{2}$ are extracted via a linear interpolation from the FLUENT three-dimensional results.

\section{Structural Dynamics Model}

Assuming the structural behavior of the windmill blade equivalent to a rotating beam, the corresponding mathematical model has been derived from the nonlinear flap-lag-torsion equations of motion presented in Hodges et al. [11]. In this representation, the blade is a cantilever, straight, slender, homogeneous, isotropic with nonuniform properties, with twist and both mass and tensile offsets. The resulting equilibrium equations are further simplified neglecting those terms considered to be of the third order with respect to the bending slope, that is an arbitrary small parameter, and not contributing to damping. Moreover, in this set of equations the radial displacement is not explicitly considered since the corresponding local tension is used. Therefore, the bending deflections are not responsible for the windmill blade axial extensions that are simply geometrical consequences of the transverse bending deflections [19]. Taking into account the previous assumptions, the dynamic system is represented by a set of coupled nonlinear integro-partial differential equations suitable for describing significant deflections $[19,20]$ of the windmill blades where the unknown state is represented by the in-plane displacement of the elastic axis, $v(x, t)$, the out-of-plane displacement of the elastic axis, $w(x, t)$, and the cross-section elastic torsion deflection, $\phi(x, t)$.

The aerodynamic load, used to couple the previous equations of motion, is based on the low-frequency approximation of two-dimensional Greenberg theory [12], leading to a simple quasi-steady strip-theory capable to take into account a pulsating free-stream for an unsteady aerodynamic loads acting on a thin airfoil. In this formulation, a first coupling with the 3D aerodynamic calculations is introduced. The local section aerodynamic parameters are evaluated from the results of the CFD calculations described in the previous section. Moreover, the aeroelastic model is completed by including the effects of the flow induced by the 
wake on the blade angle-of-attack as resulting from Eqs. (2a)(2c). Again, considering the aerodynamic 3D calculations as an experimental test bed, the changes of the angle-of-attack due to the induced velocity could be estimated from the velocity changes of the flow through the actuator disk, as reported in the previous section and in [12] and [13].

The resulting final aeroelastic system could be integrated in the space domain using the Galerkin approach adopting the non-rotating modes of the blade as shape functions. If $x$ is the vector of the modal amplitudes (Lagrangean coordinates), $\widehat{M}, \hat{C}, \widehat{K}$ are the linear time-invariant mass, damping and stiffness structural and aerodynamic contributions, and $\hat{f}$ represents the set of all nonlinear and/or time-dependent coefficient terms, then the set of the nonlinear ordinary differential equation is written as

$$
\widehat{M} \ddot{x}+\hat{C} \dot{x}+\widehat{K} x=\hat{f}(x, \dot{x}, \ddot{x}, \theta, \dot{\theta}, \ddot{\theta}, \psi)
$$

where $\psi=\Omega t$ is the azimuthal position of the blade, with $\Omega$ as the angular velocity of the rotor, and $\theta=\theta(\psi)$ is the pitch angle. Since the periodic solution is sought, then the harmonic balance approach has been used to solve these equations in the time domain.

The dynamic hub loads (root shears and moments) corresponding to the aeroelastic deformation, achieved from the previous solution, could be evaluated in the rotating frame. Then, the hub loads in the fixed frame of reference could be achieved by adding the contributions from all the blades expressed in the inertial frame of reference. If the drag, radial, and vertical shear forces at the rotor hub are denoted as $S_{x}, S_{r}$ and $S_{z}$, whereas the root bending and torque moments are $N_{F}$ and $N_{L}$ respectively, then the total forces and moments in the non-rotating frame for a $\mathrm{N}$ bladed windmill are given in [6], [23]-[26]:

$$
\begin{aligned}
& T=\sum_{m=1}^{N} S_{z m} ; \\
& \mathrm{H}=\sum_{m=1}^{N}\left(S_{r m} \cos \psi_{m}+S_{x m} \sin \psi_{m}\right) ;
\end{aligned}
$$

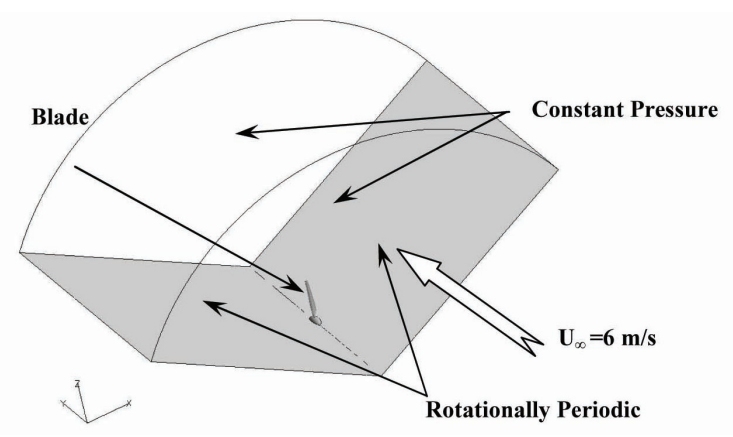

Fig. 3. Computational domain and boundary conditions.

$$
\begin{aligned}
& \mathrm{Y}=\sum_{m=1}^{N}\left(S_{r m} \sin \psi_{m}+S_{x m} \cos \psi_{m}\right) \\
& M_{x}=\sum_{m=1}^{N} N_{F m} \sin \psi_{m} ; \\
& M_{y}=-\sum_{m=1}^{N} N_{F m} \cos \psi_{m} ; \\
& Q=\sum_{m=1}^{N} N_{L m} ;
\end{aligned}
$$

\section{Numerical Results}

In this section, the results from both the aerodynamic and aeroelastic simulations are reported. In the following subsection, after a general description of the wind turbine model used in the numerical analysis, the results from the aerodynamic simulation of the rotating S809 airfoil are first outlined, then the results from to the aeroelastic simulations are presented.

\subsection{Main characteristics of the NREL S809 wind tur- bine}

The work focuses on a small wind-turbine blade designed to operate on a stall-regulated downwind wind turbine having a rated power of 20 kilowatt.

The blade geometry and dimensions were extracted from the National Renewable Energy Laboratory (NREL) experiment setup $[17,18,21,22]$, and some of these parameters are reported for completeness in Table 1 . The rotor diameter is $11.06 \mathrm{~m}$ with a root extension from the center of rotation to the airfoil transition of $0.88 \mathrm{~m}$, see Fig. 4 . The blade is twisted (root twist of $20^{\circ}$, tip twist of $-2.5^{\circ}$ about the $30 \%$ chord location) and tapered (root chord of $0.737 \mathrm{~m}$, tip chord of $0.305 \mathrm{~m}$ ) and it features a NREL S809 airfoil with a thickness ratio of $21 \%$ (see Figs. 4 and 5) [21]. The blade pitch ratio is referenced to the $75 \%$ span location, see Fig. 4 , and it is defined with respect to the $\mathrm{x}$ - and $\mathrm{y}$ axes, as shown in Fig. 5(c). The three-bladed rotor has a solidity of 0.069 and it rotates at $72 \mathrm{rpm}$ with a uniform inflow speed of $6 \mathrm{~m} / \mathrm{s}$, giving a tip-speed-ratio of 7.0. An ellipsoid of revolution is located between the blade root extension and the axis of revolution of the rotor to maintain a smooth and realistic flow field

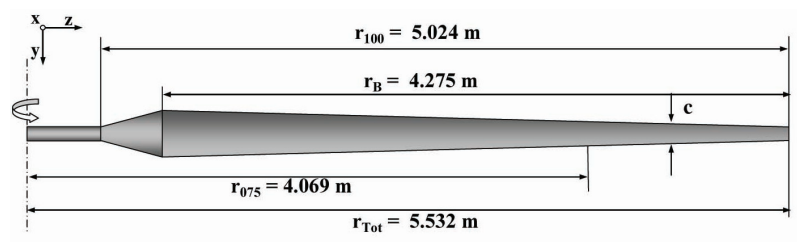

Fig. 4. Blade planform (not to scale). 


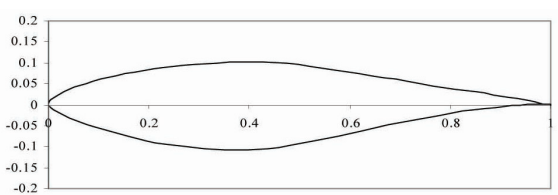

(a) Schematic view of the S809 airfoil [19].

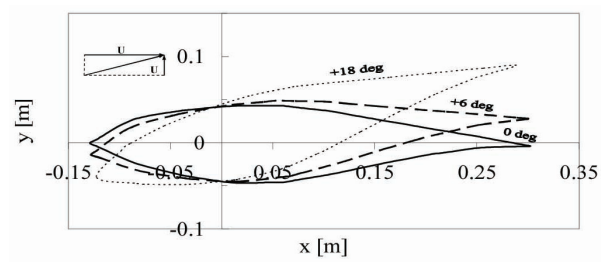

(b) Relative blade orientation for different pitch angles ( $r / R=0.75)$.

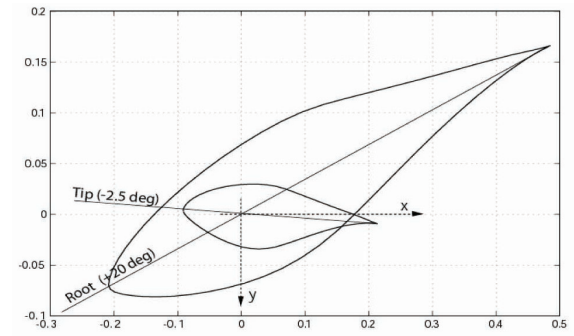

(c) Tip and root sections indicating the blade twist. (pitch angle $=0^{\circ}$ ).

Fig. 5. Schematics of the blade used in the present study

around the root region.

\subsection{Aerodynamic simulation}

This section presents the results of the aerodynamic modeling of the rotor. First, a validation study of an S809 airfoil was performed to demonstrate the validity of the CFD approach to calculate the loads on the blade structure. In Fig. 6 the pressure coefficient distribution along the chord of the wind turbine blade is presented achieved with the reference flow conditions reported in Table 2 .

The predicted $\mathrm{C}_{\mathrm{p}}$ distribution is in overall excellent agreement with the experimental data with the exception of the location at $55 \% \mathrm{c}$ where a mild flow separation is present and the CFD solution fails to predict its strength. The numerical solution shows noticeable oscillations in the $\mathrm{C}_{\mathrm{p}}$ distribution in the region ahead of $55 \% \mathrm{c}$. The oscillations are indicative of an incipient flow separation on the upper surface of the airfoil. Comparisons for the drag, lift and moment coefficient of the present work to experimental results [21] and the Wolfe and Ochs CFD simulations [22], where the $k-\varepsilon$ turbulence model was used, are presented in Table 3.

The comparison shows some discrepancy between FLUENT $^{\circledR}$ results and the experiments reported in Somers [21], especially for the drag coefficient which is $37 \%$ larger than the measured value. One source of error is the lack of detailed information on the inlet boundary conditions used in the experiment. In the present CFD simulations a uniform inlet velocity profile with a freestream velocity of $48.5 \mathrm{~m} / \mathrm{s}$ was used to match the experimental Reynolds number of $2.0 \times 10^{6}$. Additional source of error is the use of a viscous laminar flow model even at high Reynolds number where turbulence flow might significant effect, with potential effect also from turbulence boundary layer and trailing edge separation. These are all issues of great importance when looking into accuracy of the fluid-flow numerical simulations. Inlet turbulence was set at a turbulence intensity, $\mathrm{TI}=\mathrm{k} /\left(\mathrm{u}_{\infty}\right)^{2}$, of $5 \%$ and at a turbulent viscosity ratio, $\mu_{T} / \mu$, of 10 . Note that the $2 \mathrm{D}$ structured mesh consists of rectangular elements with a $\mathrm{y}^{+}$of less than 1.0 near the solid wall of the airfoil. The 3D simulations of the blade were run at five different blade pitch angles, $0^{\circ}, 2^{\circ}, 6^{\circ}, 12^{\circ}$ and $18^{\circ}$. The pitch angle is defined with respect to the airfoil at $75 \%$ of the blade radius, as shown in Fig. 7(b). For completeness and to ensure the overall similarity in order of magnitude between the turbulent and laminar results, a comparison of a laminar vs. a turbulent simulation with the Wilcox $k$ - $w$ model was run for the full

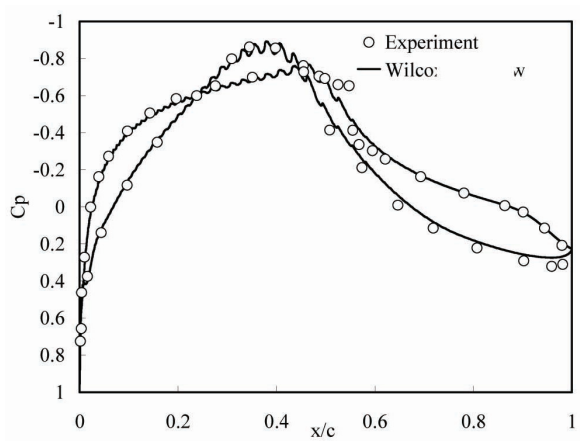

Fig. 6. Comparison of the experimental and computational pressure coefficient distribution on the airfoil for the conditions as in Wolfe and Ochs [20].

Table 3. Comparison of moments and forces for the 2D calculations.

\begin{tabular}{|c|c|c|c|c|c|c|}
\hline \hline Parameter & $\mathbf{C}_{\mathbf{l}}$ & Diff \% & $\mathbf{C}_{\mathbf{d}}$ & Diff \% & $\mathbf{C}_{\mathbf{m}}$ & Diff \% \\
\hline \hline Experiment [21] & 0.1469 & 0 & 0.0070 & & -0.0443 & 0 \\
Wolfe and Ochs [22] & 0.1324 & -10 & 0.0108 & +54 & -0.0400 & -10 \\
Present work & 0.1178 & -20 & 0.0096 & +37 & -0.0391 & -12 \\
\hline \hline
\end{tabular}


three-dimensional blade for a nominal angle of attack of 0 deg. The results are presented in Fig 6a.

While the absolute forces produced by the laminar and turbulent simulations are different, as expected, there is an overall similarity in the results, especially for the Cd. Also, it is important to notice that the methodology here proposed is valid for both flow regimes. Therefore, the $3 \mathrm{D}$ simulations were run without turbulence modeling. Also, laminar conditions might exist in some specific situations but in general it will be up to the designer to check the flow regime and run the appropriate flow model.

It is worth mentioning that other realistic computational techniques, such as the strip-theory, could be adopted in conjunction with $2 \mathrm{D}$ turbulence computations. As an example, the performance of several 2D turbulence computations at a number of axial positions along the blade could be performed and the formulation of an approximate 3D loading for the full length of the blade would then be a feasible option. This was however well beyond the scope of the present paper and it is left to the interested reader to pursue this or other viable approaches.

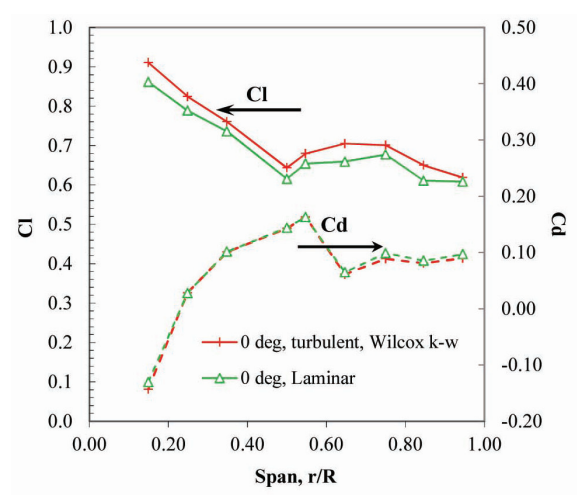

Fig. 6a. Comparison of the laminar and turbulent solutions for the full three-dimensional blade at an angle of attack of Odeg
Figure 8 shows the momentum deficit of the incoming flow once it has passed the rotor. In this isometric view the rotor is shown in full and the hub is included for clarity. The relative high-momentum in the undisturbed region away from the rotor and in the small region of undisturbed flow near the axis of rotation is clearly noticeable. The latter highlights the action of the turbine blades that are extracting linear momentum from the incoming air flow to turn it into rotor rotational momentum. The use of rotationally-periodic boundary conditions in the $3 \mathrm{D}$ simulations allowed the study of the blade-wake interaction. However, from monitoring the force time history, it appears that for a rotor angular speed of $72 \mathrm{rpm}$ and an undisturbed airspeed of $6 \mathrm{~m} / \mathrm{s}$ (tip speed ratio of 7.0) the interaction is almost negligible.

The time history of the lift, drag and moment coefficient on the blade, Fig. 9, show a Strouhal number based on a blade average chord of $0.521 \mathrm{~m}$ and an average blade velocity of $26.7 \mathrm{~m} / \mathrm{s}$ of 0.22 , corresponding to a dimensional shedding period of $0.09 \mathrm{~s}$, also confirmed by a Fast Fourier Transform analysis of the force data. This period is shorter than that of the wake shed by the preceding blades, $0.278 \mathrm{~s}$

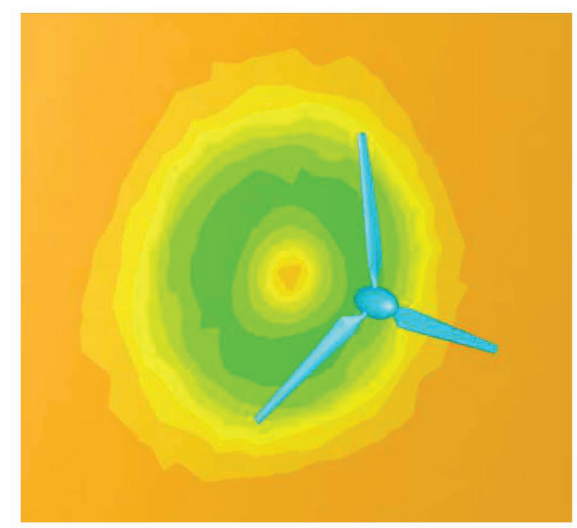

Fig. 8. Axial velocity contours.

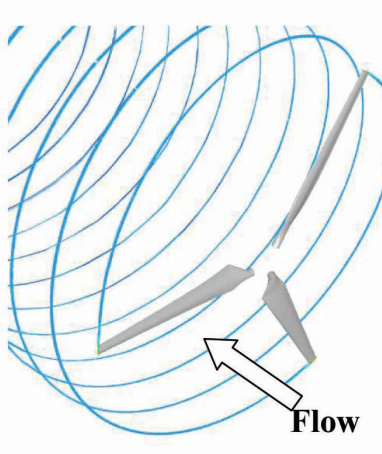

(a)

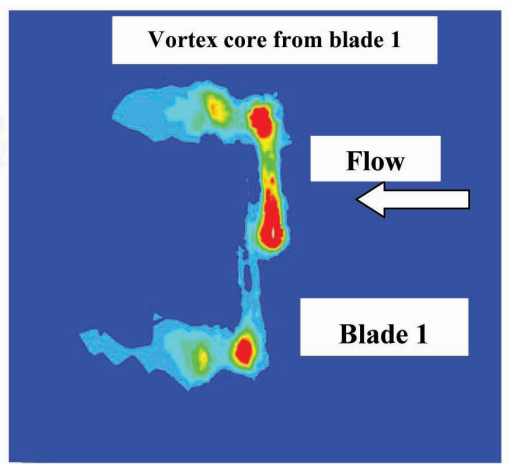

(b)

Fig. 7. (a) Tip vortices convected downstream. (b) Vorticity contours on a longitudinal plane showing the core of the wingtip vortices being con vected downstream and dissipated. Side view along blade centerline. Blade pitch angle $=6^{\circ}$. 
and it is attributable to the natural shedding frequency of the blade. Notice also that the amplitude of this oscillation is relatively small, $0.27 \%$ of the mean value for the drag and moment coefficient and $0.16 \%$ of the mean value for the lift coefficient. The blade-wake interaction is also visualized in Fig. 7(a) where the streamlines originating from the blade tips are plotted as they are convected downstream. The picture shows that the blades are well clear of the wake core produced by the preceding blade. Fig. 7(b) maps the vorticity contours on a flat plane normal to the blade axis at $\mathrm{r} / \mathrm{R}=$ 0.75. The mapping shows the vortex chores from the main blade whose cross section is visible at the center of the map. The vortex chore coming from this blade, blade 1 , is visible traveling downstream in the upper center of the figure. The vortex chore produced by the preceding blade is also visible before and after it approaches the azimuthal location of blade 1. Again, the mapping highlights the weak interaction of the blades with the wake.

The lift, and drag coefficients for the blade at different pitch angles are plotted in Fig. 10 as a function of the blade span. The coefficients are computed using the dynamic pressure at the specific blade section and the angle formed by the local velocity vector. The plot of the lift coefficient shows a consistent trend at all pitch angles of decreasing lift with span location. Also, the lift tends to decrease as the pitch angle is increased. The drag coefficient curves indicate two distinct trends depending on the pitch angle. At lower pitch angles, the drag increases steadily up to $50 \%$ of the span where it abruptly decreases, possibly indicating separation. This discontinuity decreases in intensity with increasing pitch angle and it disappears completely for a pitch angle of $12^{\circ}$ and $18^{\circ}$.

Note that, at this location for the low pitch angles there is a minor drop in lift coefficient. According to the orientation of the axes or reference, a negative drag coefficient corresponds to a "forward" thrust for the rotor, the highest power is obtained for a blade pitch angle of 6 . Lower pitch angle settings are not producing as much thrust since most of the aerodynamic force is transformed in lift which is not contributing in a major way to the production of usable power. Also note that the overall thrust is given by a combination of lift and drag. Figure 11 shows the lift-curve of the different blade sections as a function of angle-of-attack. The lift curve slope is 3.00 per radiant $(0.95 \pi / \mathrm{Rad})$ and all the blade sections show the same behavior and lift values, as expected. The lift-curve slope is much lower than the potential flow value of $2 \pi / \operatorname{Rad}$ and also of the $1.91 \pi / \operatorname{Rad}$ value calculated by Wolfe and Ochs [21].
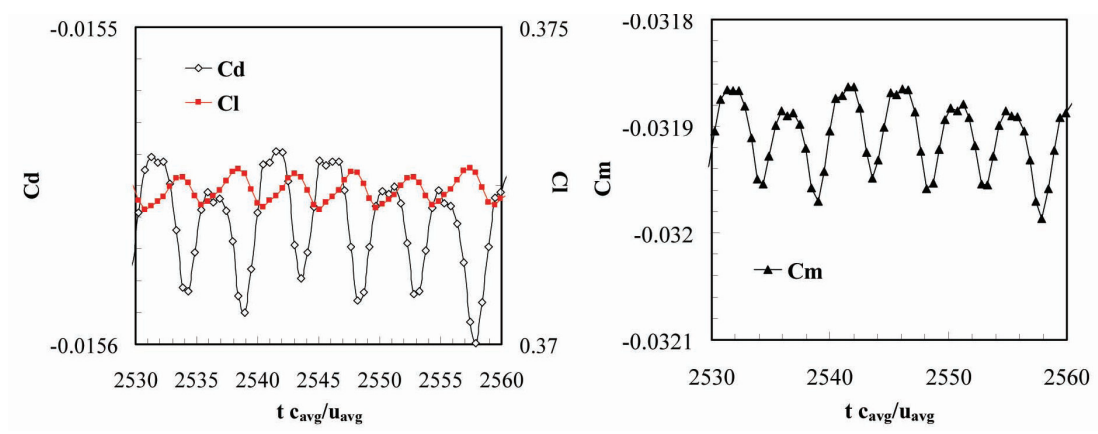

Fig. 9. Periodicity in the blade lift, drag, and moment coefficient as a function of non-dimensional time. $C_{\text {Avg }}$ and $U_{\text {Avg }}$ correspond to the arithmetic mean of the blade chord $(0.521 \mathrm{~m})$ and of the total relative velocity $(26.7 \mathrm{~m} / \mathrm{s})$. In this case the shedding period correspond to a Strouhal number of $0.22(\mathrm{t}=0.09 \mathrm{~s})$.
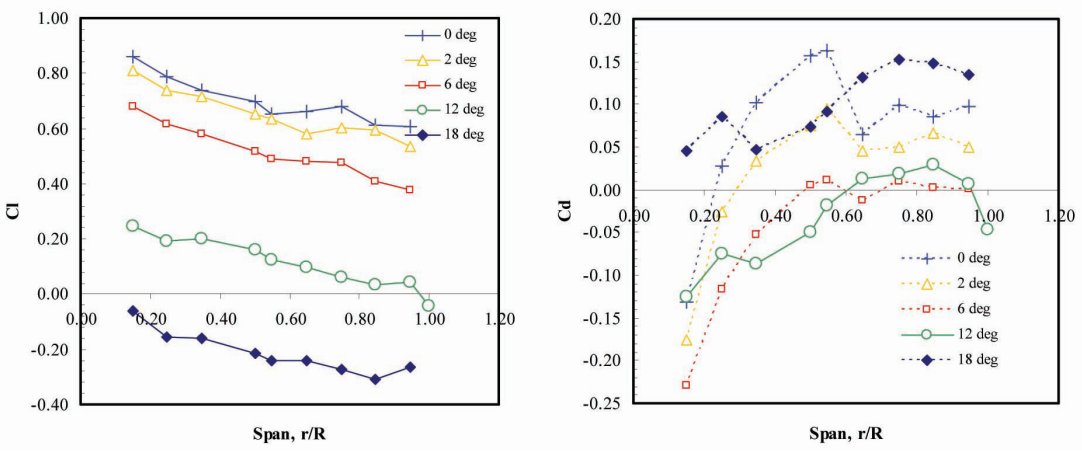

Fig. 10. Lift and drag coefficient along the blade span at different pitch angles as a function of blade radial section. 
The lower value of the $3 \mathrm{D}$ solution is due to the threedimensional effects of the blade. These effects were calculated following Bramwell [23] where the induced velocity, $U_{i}$ is calculated from

$$
\lambda_{i}^{2}+\left(\lambda_{c}+\frac{a^{\prime} \sigma(r / R)}{8}\right) \lambda_{i}-\frac{a^{\prime} \sigma(r / R)}{8}\left(\theta-\frac{\lambda_{c}}{(r / R)}\right)=0
$$

Where $\lambda_{i}=U_{i} / \omega R ; \lambda_{c}=U_{\infty} / \omega R ; \sigma=N c / \pi r ; a^{\prime}$ is the lift-curve slope (3.00/Rad in this case) and $N$ is the number

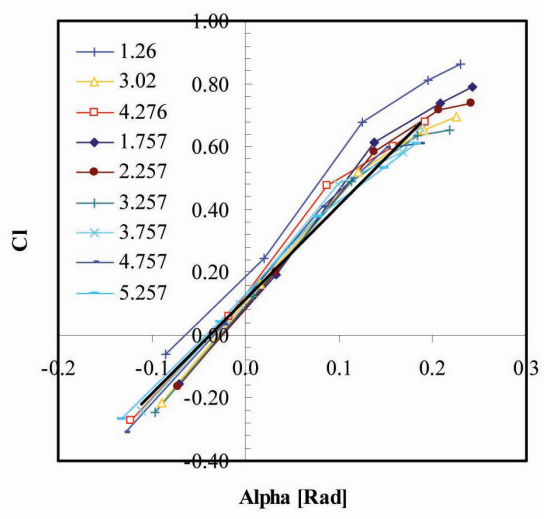

Fig. 11. Lift coefficient along the blade span at selected pitch angles as a function of blade radial section.

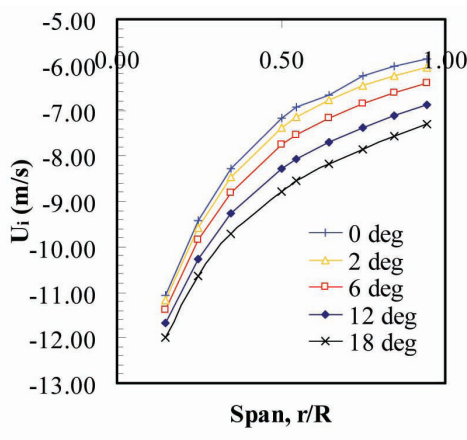

of blades of the rotor. The calculated induced velocity is shown in Fig. 12 together with the induced angle-of-attack. As expected the induced velocity is higher closer to the hub where the blade loading is generally higher. Also, the induced velocity is higher for the case with higher pitch angle and tends to decrease as the pitch angle is lowered.

The coefficients $c_{1}$ and $c_{2}$ describe the relationship between $\lambda_{i}$ and $\alpha_{\text {eff }}$ listed in Table 4 . The results obtained

Table 4. Fluent data for 3D model

\begin{tabular}{|l|l|l|l|l|}
\hline \hline $\begin{array}{l}\mathbf{r} \\
{[\mathbf{m}]}\end{array}$ & $\begin{array}{l}\mathbf{V}_{\mathbf{r}} \\
{[\mathbf{m} / \mathbf{s}]}\end{array}$ & $\begin{array}{l}\theta_{\mathrm{tw}} \\
{[\mathbf{r a d}]}\end{array}$ & $\begin{array}{l}\lambda_{\mathbf{i}}(3 \mathbf{D}) \\
{[-]}\end{array}$ & $\begin{array}{l}\boldsymbol{\alpha}_{\text {eff }} \quad(3 D) \\
{[\mathbf{d e g}]}\end{array}$ \\
\hline \hline 1.257 & 9.48 & 0.335 & 0.265 & -47.2 \\
\hline 1.757 & 13.25 & 0.182 & 0.226 & -25.0 \\
\hline 2.257 & 17.02 & 0.097 & 0.199 & -13.2 \\
\hline 3.020 & 22.77 & 0.032 & 0.172 & -4.8 \\
\hline 3.257 & 24.56 & 0.022 & 0.167 & -3.4 \\
\hline 3.757 & 28.33 & 0.000 & 0.160 & -2.6 \\
\hline 4.276 & 32.24 & -0.008 & 0.150 & 0.0 \\
\hline 4.757 & 35.87 & -0.020 & 0.145 & 1.1 \\
\hline 5.257 & 39.64 & -0.032 & 0.141 & 2.0 \\
\hline 5.532 & 41.71 & -0.038 & 0.139 & 2.5 \\
\hline \hline
\end{tabular}

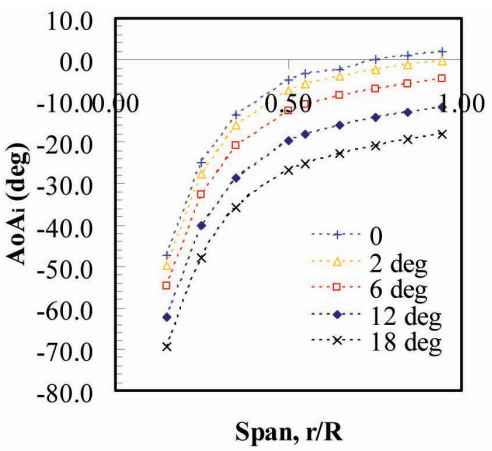

Fig. 12. Plot of induced velocity and induced angle of attack as a function of the blade span.

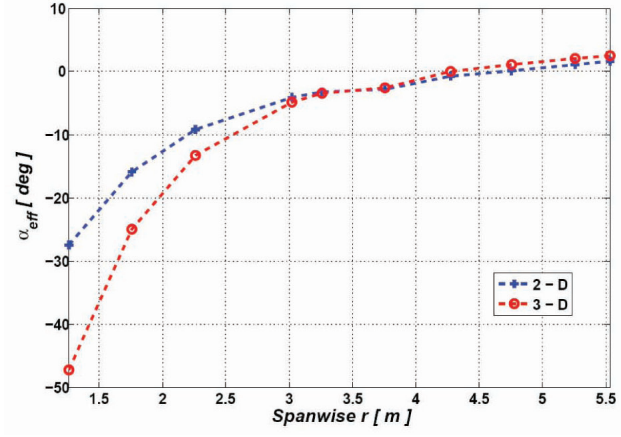

a) Effective angle-of-attack, $\boldsymbol{\alpha}_{\mathrm{eff}}$

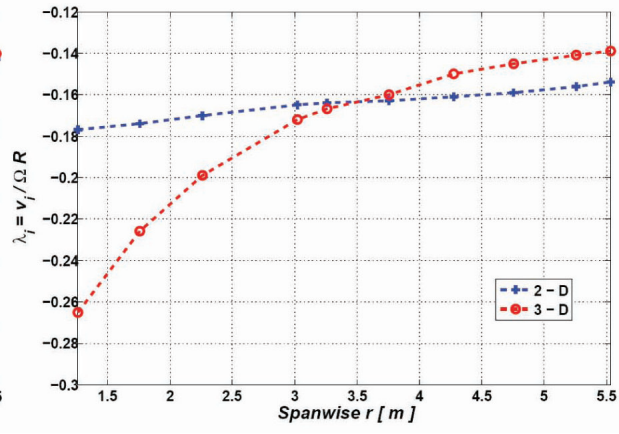

b) Inflow ratio, $\lambda^{\prime}$

Fig. 13. Comparison between the different induced velocity models. 
from the previous analysis are shown in Fig. 13; from these figures the different trend that occur for both $\lambda_{i}$ and $\alpha_{\text {eff }}$ , particularly for low values of the local spanwise position $r$, can be underlined. At the first step, the value of the effective angle-of-attack, starting from the $2 \mathrm{D}$ inflow ratio value, is calculated; The second step consist of calculation the 3D inflow ratio through the existing relationship between each couple of $\lambda_{i}$ and $\alpha_{e f f}$. This is done for every station x along the span with variable $\alpha_{e f f}$; in the last step, the difference between the $3 \mathrm{D}$ value calculated in the previous step and the first tentative $2 \mathrm{D}$ value in step one is computed: if the difference is greater than a fix tolerance, $\varepsilon=10^{-4}$, the steps are repeated in a loop, in the first step with a new value of $\lambda_{i}$ is used corresponding to the $3 \mathrm{D}$ value previously estimated.

\subsection{Structural blade characterization}

As stated in the previous section, the nonrotating modes of the blade are required in order to solve the aeroelastic system

Table 5. Comparison between the reference and the actual modal model

\begin{tabular}{|l|l|l|}
\hline \multicolumn{1}{|c|}{$\begin{array}{c}\text { Mode } \\
\#\end{array}$} & \multicolumn{1}{|c|}{$\begin{array}{c}\mathbf{f}_{\mathbf{n}} \text { Ref. [[17]] } \\
{[\mathbf{H z}]}\end{array}$} & \multicolumn{1}{c|}{$\begin{array}{c}\text { Actual } \mathbf{f}_{\mathbf{n}} \\
{[\mathbf{H z}]}\end{array}$} \\
\hline I - Flap. & 7.25 & 7.33 \\
\hline II - Flap. & 29.44 & 30.88 \\
\hline I - Lag. & 8.90 & 8.44 \\
\hline I - Tors. & Unknown & 48.00 \\
\hline \hline
\end{tabular}

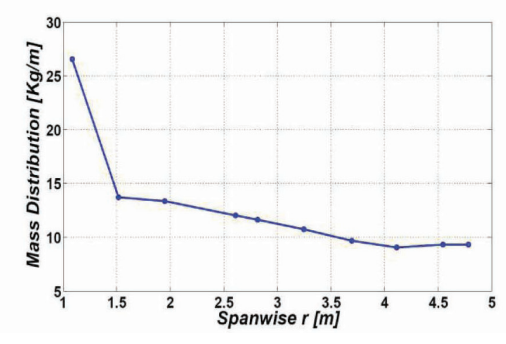

a) Mass Distribution

Fig. 14. Inertial and Stiffness distribution of the windmill blade.

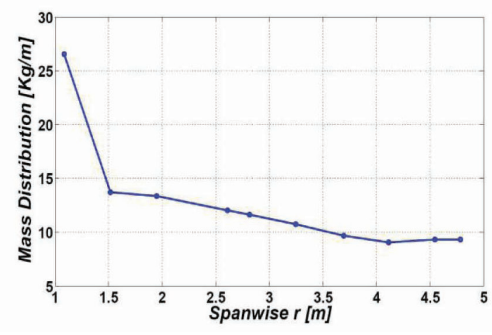

a) Flapwise displacement

Fig. 15. Comparison between the static displacements $(\psi=0)$. of equations. The flapwise and lag stiffness together with the mass distributions, required for setting up the modal model, have been evaluated using both the data reported in Giguère and Selig [17], and estimated from similar blades by using an approach based on the dynamic similitude. In Fig.14 the mass and the flapwise stiffness distribution as function of the blade span are reported, while, since no torsional stiffness data were available from [17], a typical value for this kind of blade, considered constant over the blade span, has been assumed. These structural characterizations lead to natural frequencies reported in Table 5. As one can see, the considered modal model is almost the same as the one identified in [17].

In Fig. 14 the mass and the flapwise stiffness distribution as function of the blade span are reported, while, since no torsional stiffness data were available from [17], a typical value for this kind of blade, considered constant over the blade span, has been assumed. These structural characterizations lead to natural frequencies reported in Table 5. As one can see, the considered modal model is almost the same as the one identified in [17].

\subsection{Static Aeroelastic Simulation}

Theaeroelasticsimulation has been performed considering the windmill rotating at fixed operational conditions in terms of rotational speed and blade aerodynamic incidence. This

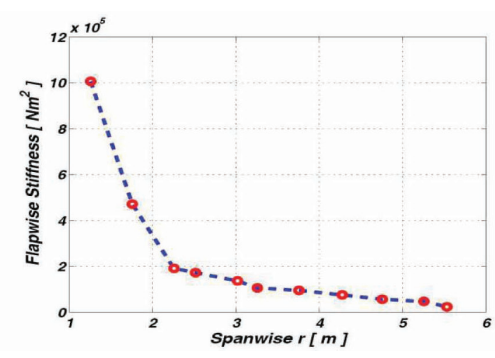

b) Out-of-Plane stiffness distribution

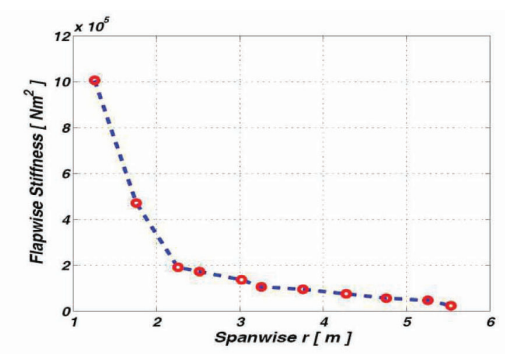

b) Lagwise displacement 
condition is equivalent to consider the windmill operating at constant, ideal, wind flow regime. The solution of the system of aeroelastic equations is carried out through harmonic balance leading to the evaluation of the elastic deformation of the hingeless blade. Herein, the aerodynamic coefficients and the inflow distributions along the blade span due to induced velocity from the $3 \mathrm{D}$ calculations are taken into account. The static component of the flap and torsional deflections, for the blade azimuthal position $\psi=0$, are presented in Figs. 15 and 16.

It is noteworthy to observe that the flap deflection occurs in the wind direction (Fig. 15a), whereas the lag occurs in the direction of the rotational velocity (Fig. 15b). By comparing these results with the ones of a rotorcraft in hover with ascension velocity equal and opposite to the one of the wind

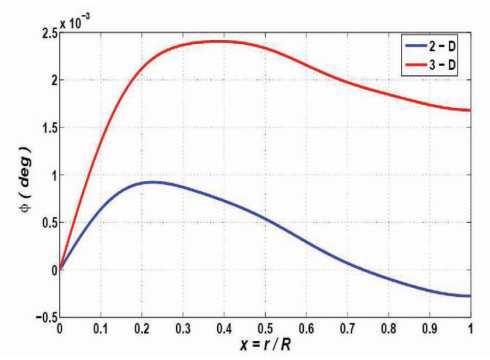

Fig. 16. Comparison between the torsional deflections $(\psi=0)$.

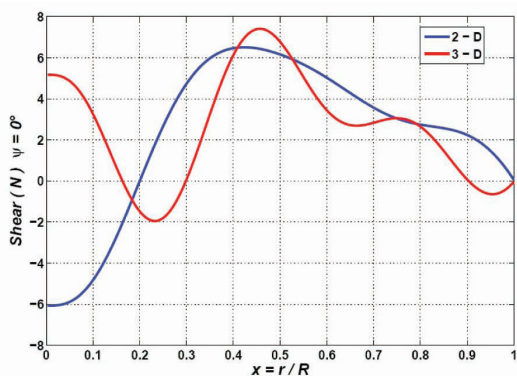

a) in-plane Shear, $S_{x}$ mill it can be noticed that the flap and lag are the opposite in directions; these results are consistent to the fact that a torque is produced at the hub of the wind mill by the interaction wind-blade system, while in the helicopter a torque at the shaft is provided by the propulsion system to generate the thrust. It is also clearly evident from these figures that the 2D model does predict lower flapwise deflections that the $3 \mathrm{D}$ model counterparts which is associated with the slightly increased effective angle of attack that is present in the 3D case, see Fig. 13a. On the other hand, the corresponding lagwise deflection is slightly lower, which might be associated with an over-estimate on the $2 \mathrm{D}$ aerodynamic loading. The different is quite small also considering also

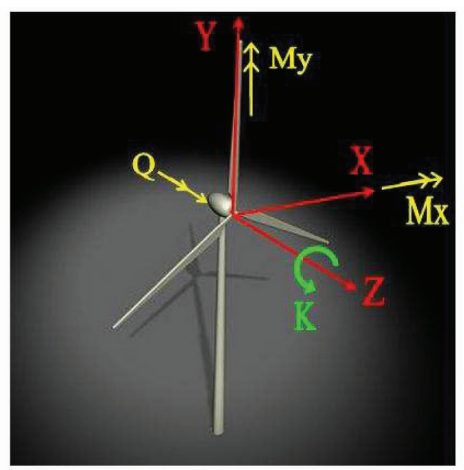

Fig. 17. Sketch of the Rotating and Fixed Reference Frames

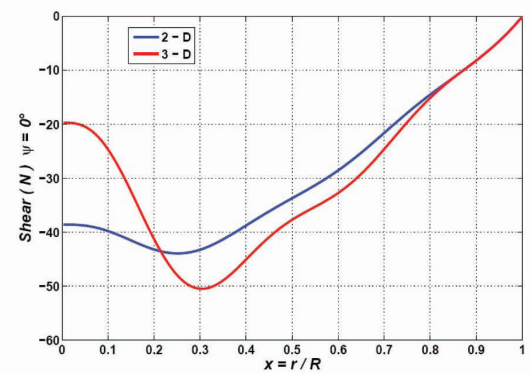

b) out-of-plane Shear $S_{z}$

Fig. 18. Comparison of the different estimates of the rotating frame reference shears $(\psi=0)$.

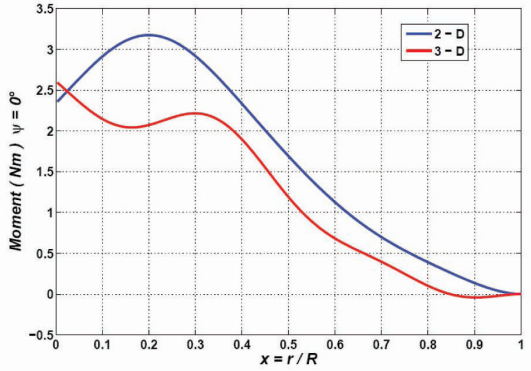

a) Lagwise bending moment, $\mathrm{N}_{\mathrm{L}}$

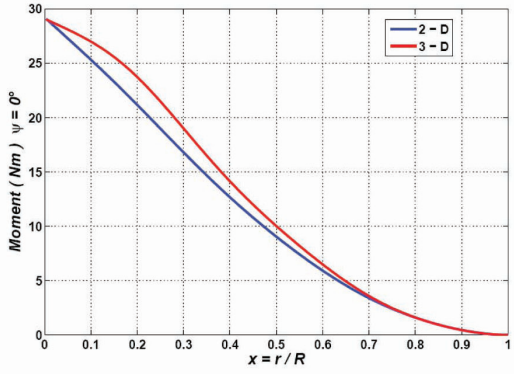

b) Flapwise bending moment, $\mathrm{N}_{\mathrm{F}}$

Fig. 19. Comparison of the different estimates of the rotating reference frame moments $(\psi=0))$. 
that in this direction the loads are one order of magnitude smaller and in the flapwise direction. The combined effect of flap and lag deflection contributes to a large discrepancy in the torsional direction, as it is displayed in Fig. 16. Clearly the inherent flexibility of the rotor combined with the complex aerodynamic characteristics in the spanwise direction creates some complexities in the deformation field and it is quite challenging to fully interpret. It is also possible to estimate the shear and moments along the span of the blade in the undeformed rotating reference frame attached to the blade, as reported in Fig. 17.

The comparison between the dynamic loads as function of the blade span, considered at $\psi=0$, are reported in Figs. 18 and 19 where the different evaluations of the dynamic

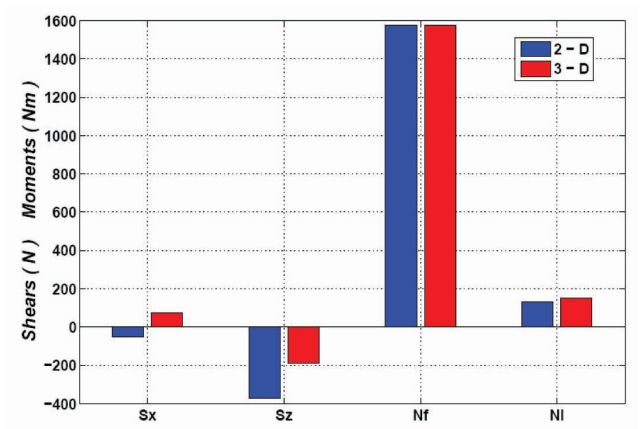

Fig. 20. Comparison of the different static hub load estimates in the rotating reference frame.

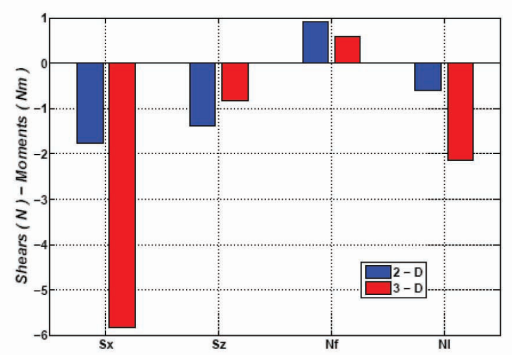

a) $\cos (1 / \mathrm{rev})$ rotating shears and moments, are depicted. Moreover, the different hub load estimates, always in the rotating reference frame, are reported in Figs. 20 through 22. From Fig. 20, only a remarkable difference in the static out-of-plane shear is reported, whereas a completely different behavior, but with much smaller amplitudes, of the dynamic elastic reactions (acting at 3/rev and 5/rev harmonics), is evident in Figs. 21 and 22 , respectively.

When considering the resulting loads acting in the fixed reference frame, the convention reported in Fig. 17 are used. For the positive values of the hub dynamic loads: the thrust, $\mathrm{T}$, is developed by the rotor acting in the orientation of the wind; the torque, $\mathrm{Q}$, (responsible for the power generation of the wind turbine) has the orientation of $\Omega ; \mathrm{H}$ and $\mathrm{Y}$ are the shears acting in the $x$ and $y$ reference directions, respectively; and, finally, $\mathrm{M}_{\mathrm{x}}$ and $\mathrm{M}_{\mathrm{y}}$ are the bending moments in the $\mathrm{x}$ and $\mathrm{y}$ axis, respectively. The aeroelastic loads evaluated in the fixed reference frame excite the windmill structure at discrete frequencies that are the number of blades of the rotor times the rotating angular frequency (see e.g. [20] through [22], [27]) plus the static component.

The different evaluations of the static loads are reported in Fig. 23, whereas Fig. 24 refers to the 3/rev harmonics loads.

For the lateral equilibrium, the rotor forces $\mathrm{H}, \mathrm{Y}$ and the moments $M_{x}, M_{y}$ should be approximately zero; indeed this is the case as represented in Fig. 23. It appears that the $2 \mathrm{D}$

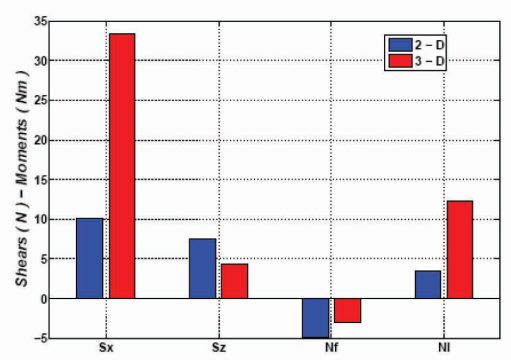

b) $\sin (1 / \mathrm{rev})$

Fig. 21. Comparison of the different harmonic hub load estimates in the rotating reference frame.

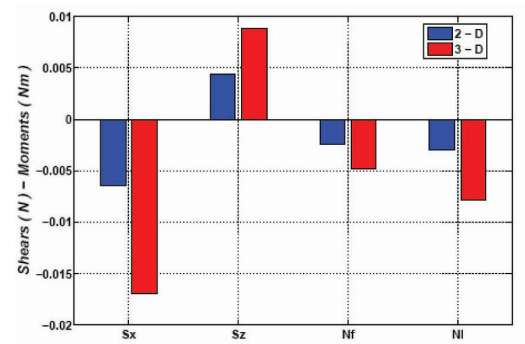

a) $\cos (3 / \mathrm{rev})$

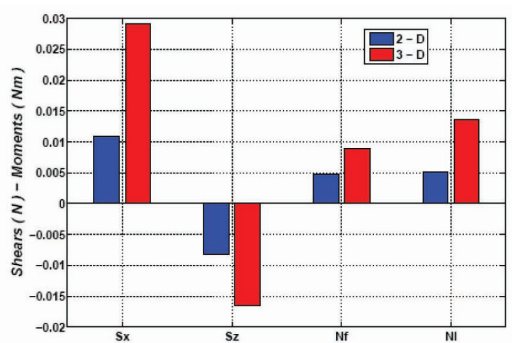

b) $\sin (3 / \mathrm{rev})$

Fig. 22. Comparison of the different harmonic hub load estimates in the rotating reference frame. 
and 3D aerodynamic models predict dissimilar results, in particular between the force $\mathrm{T}$ and moment $\mathrm{Q}$, being the value of the $2 \mathrm{D}$ model greater than the one of the in $3 \mathrm{D}$ model. The different evaluation of the dynamic loads leads to an over estimate of the $2 \mathrm{D}$ static thrust with respect to the one predicted by a $3 \mathrm{D}$ analysis. This fact could be of great interest in designing the windmill tower since the resulting bending moments, evaluated at the basement of the tower, are significant different, i.e., the 3D simulation provides lesser stressed tower structure than the 2D analysis. Furthermore, the $3 \mathrm{D}$ analysis predicts a value of moment $\mathrm{Q}$ that is greater than the correspondent $2 \mathrm{D}$ value, resulting in the capability of the windmill turbine to extract higher power.

\section{Conclusions}

Unsteady aerodynamics and aeroelastic characteristics of a medium-sized wind-turbine blade operating under ideal conditions have been considered. The tapered/twisted blade was designed to operate on a stall-regulated downwind wind turbine originally studied in an experiment setup at the NREL. The structural dynamics of the flexible wind-turbine blade has been described by a nonlinear flap-lag-torsion slenderbeam differential model. The aerodynamic quasi-steady forcing terms needed for the aeroelastic governing equations

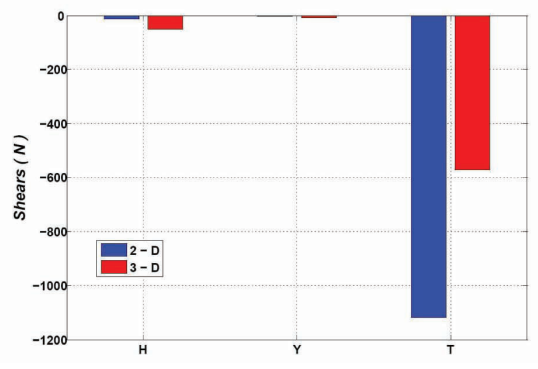

a) Shears have been predicted through a strip-theory based on a simple 2D model, whose pertinent aerodynamic coefficients have been obtained using CFD. Aerodynamic loads are computed by CFD and analytical techniques. Turbulence effects in the 2D simulations run for validation purposes were accounted by the Wilcox $k-w$ model. In this first approximation of the aerodynamic loading, the 3D CFD simulations were run using the unsteady laminar Navier-Stokes equations solved via a commercial software, FLUENT $^{\circledR}$. Five blade pitch angles were considered and their aerodynamic characteristics compared. The $6^{\circ}$ pitch angle was found to be producing the highest power setting due to the relatively high (propulsive) drag force. For pitch angles higher than $6^{\circ}$ the blade showed an increased drag coefficient indicating less-than-optimal performance. The interaction of the rotor blades with their own wake was found to be negligible for the operating conditions under consideration due to the convective effect of uniform airflow. The resulting unsteady hub loads are achieved by a first space integration of the aeroelastic equations by applying the Galerkin's approach and by a time integration using a harmonic balance scheme. Two and three dimensional computations for the unsteady aerodynamic loads, the flap, lag and torsional deflections, and the forces and moments applied at the hub of rotor along with aeroelastic simulations are illustrated. Structural results have shown that the responses to the $2 \mathrm{D}$ and $3 \mathrm{D}$ aerodynamic models produce dissimilar results, therefore stressing the importance to keep the dimensionality effects

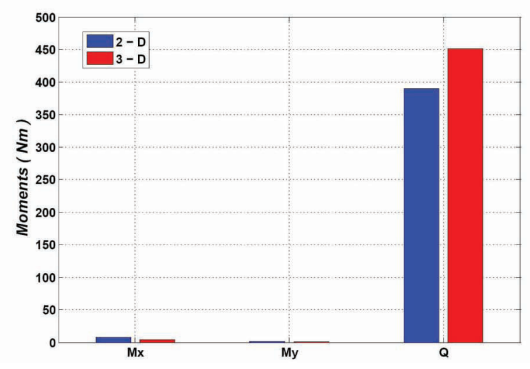

b) Moments

Fig. 23. Comparison of the different static hub load estimates in the fixed reference frame.

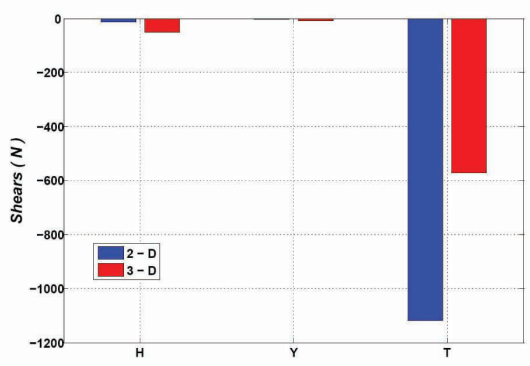

a) $3 /$ rev Shears

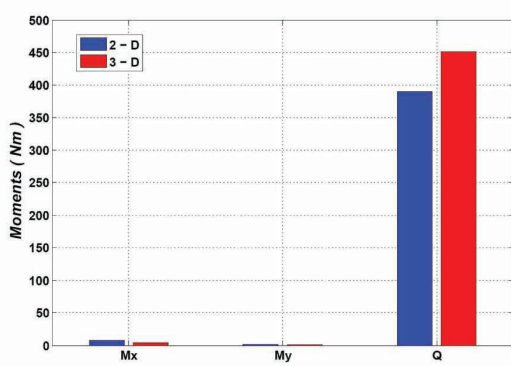

b) $3 /$ rev Moments

Fig. 24. Comparison of the different harmonic hub load estimates in the fixed reference frame. 
into consideration if quantitative results are expected from the simulations. To this point the authors acknowledge that turbulence models are very problematic for these flows, as the flow typically transitions at some point on the airfoil, and none of the usual turbulence models currently available handle very well the adverse pressure gradient, that occur on the aft of the airfoil, very well. Therefore experimental data will always be needed to back up computational predictions. Clearly neglecting flow separation along with turbulence, especially in 3D simulations can lead to inaccurate results. In particular, 3D analysis predicts a value of a torque, and consequently the power output, that is a conservative estimation of the energy that can be extracted from the wind. However, since the main goal of this paper is not to provide an highly accurate aerodynamic model of the wind turbine during operational condition, but rather to demonstrate the proof-of-concept of an innovative way to perform reliable and fast flow-structure simulations for aeroelastic design purposes, the assumptions made are reasonable and can be easily extended to more realistic aerodynamic models. Also the design of the wind turbine tower could benefit from the results of the $3 \mathrm{D}$ simulations performed since a lesser stressed tower is predicted. Future work will focus on the turbulence effects on the 3D simulations using unsteady RANS equations and DES techniques, and on the inclusion of an atmospheric boundary layer and asymmetric hub loading.

\section{Acknowledgments}

The authors would like to thank the Metropolitan Development Association of Syracuse and Central New York, Inc and The National Science Foundation, grant NSF-CMMI-1031036, for providing partial support for this research. Also, partial support for the present work was provided by the University of Rome, "La Sapienza" through the project "Progetto Giovani Ricercatori: Dinamica ed Aeroelasticita dei Rotori di Elicottero".

\section{References}

[1] Burton, T., Sharpe, D., Jenkins, N., and Bossanyi, E., Wind Energy Handbook, $1^{\text {st }}$ Edition, John Wiley \& Sons, Ltd., Chichester, 2001, ISBN: 0-471-48997-2

[2] Manwell, J. F., McGowan, J.G., Rogers, A.L. "Wind Energy Explained", $1^{\text {st }}$ edition, John Wiley \& Sons, Ltd., Chichester, 2002, ISBN 0-471-49972-2.

[3] Eggleston, D.M., Stoddard, F.S. "Wind Turbine Engineering Design", Van Nostrand Reinhold, New York,
1987, p. 307.

[4] Germanischer Lloyd, Rules and regulations, VI - Nonmarine Technology, Part 1 - Wind Energy (Hamburg, 1993).

[5] L. Librescu, P. Marzocca, and W. A. Silva, "Aeroelasticity of 2-D lifting surfaces with time-delayed feedback control," Journal of Fluids and Structures, Vol. 20, No. 2, 2005, pp. 197215.

[6] A. Behal, V. M. Rao, P. Marzocca, and M. Kamaludeen, "Adaptive Control for a Nonlinear Wing Section with Multiple Flaps," Journal of Guidance, Control, and Dynamics, Vol. 29, No. 3, 2006, pp. 744-748.

[7] Leishman, J.G., "Principles of Helicopter Aerodynamics", $1^{\text {st }}$ Edition, Cambridge University Press, 2000, ISBN 0-52152396-6.

[8] Leishman, J. G., "Challenges in modeling the Unsteady Aerodynamics of Wind Turbines,", AIAA 2002-0037, $21^{\text {st }}$ ASME Wind Energy Symposium and $40^{\text {th }}$ AIAA Aerospace Sciences Meeting, Reno, NY, 2002.

[9] Politis, E.S., Nikolaou, I.G., Chaviaropoulos, P.K., Bertagnolio, F., Sørensen, N.N., and Johansen, J., "KNOWBLADE Task-4 report; Navier-Stokes Aeroelasticity", Risø-R-1492 (EN), Risø National Laboratory, Roskilde, Denmark, 2005.

[10] Wilcox, D.C., “Turbulence Modeling for CFD”, DCW Industries, Inc., La Canada, California, 1998.

[11] Hodges, D. H., and Dowell, E. H., "Nonlinear Equation for the Elastic Bending and Torsion of Twisted Non Uniform Rotor Blades," NASA TN D-7818, 1974.

[12] Greenberg, J.M., "Airfoil in Sinusoidal Motion in a Pulsating Stream," NACA TN-1326, 1947.

[13] Theodorsen, T., "General Theory of Aerodynamic Instability and the Mechanism of Flutter," NACA Report No.496, 1935.

[14] Fluent, Inc., FLUENT User Guide, September 29, 2006.

[15] Stepniewski, W.Z., Keys, C.N. "Rotary - Wing Aerodynamics”, Dover Publications, Inc. , New York, 1984.

[16] Johnson, W., "Helicopter Theory", Dover Publications, 1980.

[17] Giguère, P., and Selig, M. S., "Design of a Tapered and Twisted Blade for the NREL Combined Experiment Rotor", NREL/SR-500-26173, Golden, CO: National Renewable Energy Laboratory, 1999.

[18] Butterfield, C.P., Musial, W.P., Simms, D.A., "Combined Experiment Phase I Final Report", NREL/TP257-4655, 1992, Golden, CO: National Renewable Energy Laboratory.

[19] Hodges, D.H., and Ormiston R.A., "Stability of Elastic Bending and Torsion of Uniform Cantilever Rotor Blades in Hover with Variable Structural Coupling," NASA TN D-8192, 1976. 
[20] Friedmann, P.P., "Helicopter Rotor Dynamics and Aeroelasticity: Some Key Ideas and Insights," Vertica, Vol.14, No.1, pp.101-121, 1990.

[21] Somers, D. M., "Design and Experimental Results for the S809 Airfoil,", Technical Report, Airfoils, Inc., State College, PA, 1989.

[22] Wolfe, W.P., Ochs, S.S., "CFD Calculations of S809 Aerodynamic Characteristics”, AIAA Paper 97-0973, 1997.

[23] Bramwell, A.R.S., "Helicopter Dynamics", Butterworth -Heinemann Ltd, June 1, 1976.

[24] Bir, G.S., "Structural Dynamics Verification of Rotorcraft Comprehensive Analysis System (RCAS)", NREL/ TP-500-35328, 2005, Golden, CO: National Renewable
Energy Laboratory.

[25] Bielawa, R.L., "Rotary Wing Structural Dynamics and Aeroelasticity," AIAA Education Series, AIAA, 1992.

[26] Coppotelli, G., and Cellini, F., "A Rotorcraft Trim Procedure for the Prediction of Fuselage Vibrations," Advances in Engineering Research, Vol. 4, Nova Science Publisher, NY, USA, pp. 235-257. ISBN: 9781621006954

[27] Balis Crema L., Coppotelli, G., and Grappasonni, C., "On the Effects of Structural Modifications in the Large Wind Turbine Dynamics," Proceedings of the 52nd AIAA/ASME/ ASCE/ASC Structures, Structural Dynamics and Materials Conference, Denver (CO) - USA, Denver (Co) - USA, 2011. 